\title{
LINEARLY CONSTRAINED MEG BEAMFORMERS FOR MVAR MODELING OF CORTICAL INTERACTIONS
}

\author{
Hua Brian Hui, Richard M. Leahy
}

Signal and Image Processing Institute, University of Southern California, Los Angeles, CA 90089

\begin{abstract}
Among the many methods for modeling cortical interactions using EEG and MEG data, Multivariate Autoregressive(MVAR) functional connectivity measures have the advantage of providing parametric directional and frequency specific information. While MVAR models have been successfully applied to depth electrode data, they are more difficult to use with external EEG and MEG data since they are not robust to the crosstalk between cortical regions that may arise because of the limited spatial resolution of EEG/MEG inverse procedures. Here we describe a modified beamforming approach for processing EEG/MEG data, designed to eliminate cross-talk between cortical regions. The output of the beamformer is then used to estimate the coefficients of an MVAR model of cortical interactions. We illustrate this method using simulated dynamic MEG data.
\end{abstract}

\section{INTRODUCTION}

Modeling distributed dynamical interactions or functional connectivity between cortical regions is a key issue in understanding neural interactions in the human brain. Many different methods have been proposed to model functional connectivity including spectral coherence and phase synchronization. These methods characterize the strength of the interaction, but provide no directional information and are typically nonparametric so have relatively poor frequency resolution. Multivariate autoregressive (MVAR) time series methods adopt a parametric approach giving improved frequency resolution and are also able to establish the directionality of interactions using the concept of Granger causality [1] [2] [3].

MVAR methods have been used to characterize functional connectivity from depth electrode measurements [4]. While these methods extend in principle to external EEG and MEG measurements, their utility is limited by their sensitivity to linear cross-talk. If applied to raw EEG and MEG data, MVAR models would be unable to distinguish between true regional connectivity within the brain and the simple linear cross-talk between sensor measurements that results from the broad spatial sensitivity of the sensors. To better localize cortical activity, inverse procedures can be used to compute images of

This work was supported by NIBIB under Grant No R01 EB002010. current density in the cerebral cortex. Inverse methods include dipole fitting, beamforming, and cortically constrained imaging. Of these, linearly constrained minimum variance (LCMV) beamforming and linear regularized minimum norm imaging are the most widely used. The limited resolution of minimum norm imaging leaves substantial cross-talk between regions, even after reconstruction, as we demonstrate below. Consequently MVAR models may not generally perform well when used in conjunction with linear imaging methods, although examples of their combined use can be found in the literature [5].

LCMV beamformers have higher resolution than minimum norm imaging, at least in the case where cortical sources are focal. However, the design criterion of minimizing output power can result in signal cancellation between interacting regions when signals exhibit coherent behavior. It follows that standard LCMV beamformers can produce partial cancellation of the coherent signals that we are looking for and hence are not suitable for use in combination with MVAR modeling; again we demonstrate this below. Here we describe a modified LCMV approach in which we use additional nulling constraints to prevent cross-talk between regions. Furthermore we use a set of eigenvector constraints to make the methods robust to specification of the precise location and extent of the cortical sources of interest. We demonstrate this procedure in application to simulated MEG data.

\section{METHODS}

\subsection{The MVAR Model}

The MVAR model is a straightforward extension of the univariate autoregressive time series model to multiple time series, which incorporates interactions between the time series. With $\mathbf{x}(t)=\left[x_{1}(t), \ldots, x_{p}(t)\right]^{T}$ representing a $\mathrm{p}$-dimensional time series, the MVAR model can be expressed as:

$$
\mathbf{x}(t)+\mathbf{A}(1) \mathbf{x}(t-1)+\cdots+\mathbf{A}(m) \mathbf{x}(t-m)=\mathbf{n}(t)
$$

Here $\mathbf{n}(t)=\left[n_{1}(t), n_{2}(t), \ldots, n_{p}(t)\right]^{T}$ is a zero mean uncorrelated noise vector with covariance matrix $\boldsymbol{\Sigma}$ and the $\mathbf{A}(k)$ 's are the $p \times p$ AR coefficient matrices. The coefficients for a particular data set are estimated by solving a multivariate version of the Yule-Walker equations.

After fitting the MVAR model, multivariate spectral analysis can be performed by transforming (1) into the frequency 
domain [3]:

$$
\mathbf{x}(f)=\mathbf{A}^{-1}(f) \mathbf{n}(f)=\mathbf{H}(f) \mathbf{n}(f)
$$

Where vector $\mathbf{x}(f)$ represents the Fourier transform of the time series and $\mathbf{H}(f)$ is a $p \times p$ transfer function matrix,

$$
\mathbf{H}(f) \equiv[\mathbf{A}(f)]^{-1} \equiv\left[\sum_{k=0}^{m} \mathbf{A}(k) e^{-2 \pi i k f}\right]^{-1}
$$

Using this general form, a number of different functional connectivity metrics can be computed: Granger Causality [4], the Directed Transfer Function [2] , and Partial Directed Coherence [3].

The Directed transfer function (DTF) [2] and Partial Directed Coherence (PDC) [3] are functional connectivity measures which are frequency dependent matrix functions of the transfer function matrix. The $(i, j)$-th entry of the matrix represents a measure of the interaction from source $i$ to source $j$. They are defined using the elements of $\mathbf{H}(f)$ or its inverse $\mathbf{A}(f)$ as follows:

$$
\operatorname{DTF}_{i j}^{2}(f)=\frac{\left|H_{i j}(f)\right|^{2}}{\sum_{k=1}^{p}\left|H_{i k}(f)\right|^{2}} \quad \operatorname{PDC}_{i j}^{2}(f)=\frac{A_{i j}^{2}(f)}{\sum_{k=1}^{p} A_{k j}^{2}(f)}
$$

\subsection{The Standard LCMV Beamformer}

The LCMV beamformer is a spatial filtering technique first applied in radar and sonar signal processing [6] and has been widely used in the analysis of EEG and MEG data [7]. LCMV spatial filters minimize the variance or power at the filter output subject to passing signals from a cortical region of interest with unit gain. In this way the LCMV beamformer can adaptively select the coefficients of the filter to reduce the noise in the output that is independent of the signal of interest, while passing the desired signal through the filter without attenuation. The LCMV beamformer design problem is formulated as:

$$
\min _{\mathbf{w}\left(q_{i}\right)} \operatorname{tr}\left[\mathbf{w}^{T}\left(q_{i}\right) \mathbf{C}(\mathbf{x}) \mathbf{w}\left(q_{i}\right)\right] \quad \text { subject to } \quad \mathbf{w}^{T}\left(q_{i}\right) \mathbf{g}\left(q_{i}\right)=1
$$

where $\mathbf{C}(\mathbf{x})$ denotes the spatial covariance matrix of the data and $\mathbf{g}\left(q_{i}\right)$ denotes the sensitivity or gain vector for a unit current dipole source centered at the cortical region of interest.

The LCMV beamforming method has an inherent signal cancellation problem: when a source correlated with the signal of interest is present, the filter will adapt to use the correlated signal to cancel some fraction of the signal of interest [7]. Consequently, the beamformer in its standard form is of limited use in investigating cortical interactions, where correlation between regions is assumed to be present.

\subsection{A Nulling Beamformer to Avoid Signal Cancellation}

To eliminate the cancellation effect, one has to make sure that the filter output at one source location will not be affected by the other sources. This can be achieved by using additional nulling constraints, i.e. the zero response conditions at those locations have to be satisfied:

$$
\mathbf{w}^{T}\left(q_{i}\right) \mathbf{g}\left(q_{j}\right)=0 \quad \text { with } \quad j \neq i
$$

Combining these nulling constraints with the unit response condition of the traditional LCMV, the beamformer design problem can be written using the gain matrix $\mathbf{G}=\left[\mathbf{g}\left(q_{0}\right) \cdots \mathbf{g}\left(q_{s}\right)\right]$ as:

$$
\min _{\mathbf{w}\left(q_{i}\right)} \operatorname{tr}\left[\mathbf{w}^{T}\left(q_{i}\right) \mathbf{C}(\mathbf{x}) \mathbf{w}\left(q_{i}\right)\right] \quad \text { subject to } \quad \mathbf{w}\left(q_{i}\right)^{T} \mathbf{G}=\mathbf{f}_{i}^{T}
$$

where $\mathbf{f}_{i}$ represents the $i$-th column vector of an identity matrix, and $s$ is the number of sources.

This minimization problem is readily solved using Lagrange multipliers [7]:

$$
\mathbf{w}\left(q_{i}\right)=\mathbf{C}^{-1}(\mathbf{x}) \mathbf{G}\left[\mathbf{G}^{T} \mathbf{C}^{-1}(\mathbf{x}) \mathbf{G}\right]^{-1} \mathbf{f}_{i}
$$

The output of this new beamformer method is explicitly constrained to avoid cross-talk or signal cancellation between each of the $s$ cortical regions of interest and should therefore be suitable for use to assess functional connectivity using the MVAR model.

\subsection{Eigenvector Constraints for Distributed Sources}

Our proposed beamformer method works well for point sources on the cortex. However, in reality, broad cortical regions can be active in response to specific sensory or cognitive challenges. This raises the possibility of self cancellation if the actual location and extent of the source produces a signal at the EEG/MEG array that is substantially different from that of the point source model. To overcome this problem, we use eigenvector constraints [6].

The idea is to represent the potential range of measurements at the array for a distributed source by first generating the forward gain matrix for all source locations in a tesselated cortical patch that represents the region of interest. Instead of placing a point constraint at each location, we use a limited number of constraints based on a least squares approximation to the desired response:

$$
\min _{\mathbf{w}\left(q_{i}\right)}\left\|\mathbf{w}^{T}\left(q_{i}\right) \mathbf{A}-\mathbf{r}_{d}\right\|^{2}
$$

where $\mathbf{A}=\left[\mathbf{g}\left(q_{1}\right) \cdots \mathbf{g}\left(q_{k}\right)\right]$ is the lead field matrix for all $k$ locations on a single patch source, $\mathbf{r}_{d}$ is the desired response vector for these $k$ locations, which is a vector of one for the region of interest and all zeroes for the other regions.

To limit the number of constraints to a small number $l$ for each patch, a rank $l$ approximation of $\mathbf{A}$ is used from its singular value decomposition (SVD) $\mathbf{A}=\mathbf{U} \boldsymbol{\Sigma} \mathbf{V}^{\dagger}$ :

$$
\mathbf{A}_{L}=\mathbf{U}_{L} \boldsymbol{\Sigma}_{L} \mathbf{V}_{L}^{\dagger}
$$

$\Sigma_{L}$ is an $l \times l$ diagonal matrix with the $l$ largest singular values of $\mathbf{A}$, and $\mathbf{U}_{L}$ and $\mathbf{V}_{L}$ are matrices containing the $l$ singular vectors of $\mathbf{U}$ and $\mathbf{V}$ corresponding to the singular values in $\boldsymbol{\Sigma}_{L}$.

By replacing $\mathbf{A}$ in (9) with $\mathbf{A}_{L}$, the solution of the minimization problem is:

$$
\mathbf{w}^{T}\left(q_{i}\right) \mathbf{U}_{L}=\mathbf{r}_{d}^{\dagger} \mathbf{V}_{L} \boldsymbol{\Sigma}_{L}^{-1}
$$

By applying these constraints to each of the $s$ regions of interest, a group of $s \times l$ constraints are generated for designing 
the beamformer; again the weights of the spatial filter are obtained using Lagrange multipliers. A different beamformer is designed for each region of interest, and the outputs of the $s$ beamformers serve as the input for the MVAR model.

\subsection{Tests for Significance}

To investigate specific cortical interactions it is common to integrate the DTF over a frequency band of interest; in the following simulation we investigate an approximate beta band by integrating from $12-30 \mathrm{~Hz}$. This results in a single $s \times s$ DTF matrix which represents an estimate of the strength of interaction between each region in the beta band. To assess the significance of these interactions, i.e. whether the detected regional interactions could have occurred by chance, we use a permutation test [4]. In this work we assume that the data consist of multichannel MEG measurements corresponding to multiple repetitions (or "epochs") of the same condition. Data covariances are computed over short time intervals averaged over multiple epochs. The exchangeability condition required to use the permutation test is as follows: the signals from each region are independent under the null hypothesis of no interactions between these regions. Consequently, we can learn the null distribution for the DTF by randomly permuting the epoch indices separately for each region of interest and repeating the MVAR analysis. We can then use this null distribution to threshold the computed DTF measures at a chosen significance level. Furthermore, it is straightforward to use the permutation test to control for errors in multiple hypothesis testing (one per matrix element) by using the maximum distribution to set the threshold. Note that this permutation test will not work if the source signals are estimated from methods where there is cross-talk between sources. The reason is that the permutations will destroy this cross-talk and lead to the possible interpretation of nonzero DTF's, which are the result of the cross-talk, as significant cortical interactions.

\section{COMPUTER SIMULATION}

To investigate the performance of the nulling beamformer in conjunction with the MVAR model we simulated MEG data as follows. Time series were simulated to represent 5 cortical regions as shown in Fig. 1 (left). These locations are believed to be the active areas involved in attentional control of visual processing [8]. The time series were generated using a 5 dimensional MVAR model with coefficients chosen to produce significant interactions in the beta band (approx $15 \mathrm{~Hz}$ ): $1 \rightarrow 2$ (from region 1 to region 2 ), $1 \rightarrow 3,1 \rightarrow 4$, $4 \rightarrow 5,5 \rightarrow 4$ and $1 \rightarrow 5$. For each region we defined a cortical patch with sizes as follows: $3.24,3.54,1.7,2.51$ and $1.63 \mathrm{~cm}^{2}$ for regions 1 to 5 , respectively. One time series was assigned to each patch and then MEG data were modeled using a lead field matrix based on a spherical head model. The MEG sensors were configured to simulate the 275 magnetometers in the CTF whole head system. We generated 100 epochs of MEG data using the MVAR time series and used this to compute inverse solutions and MVAR model parameters as described below.
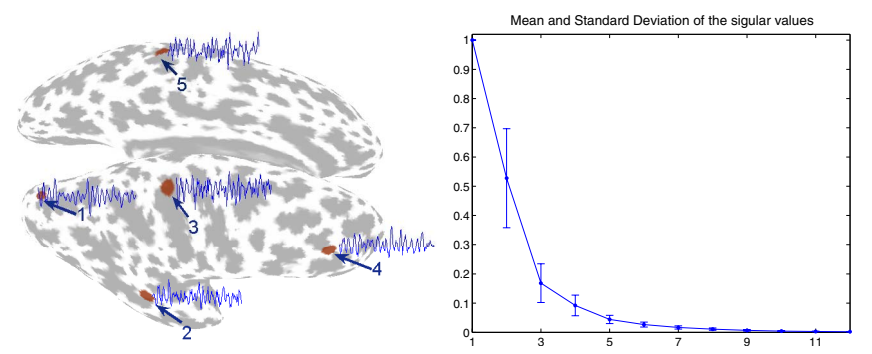

Fig. 1. (Left) Simulated cortical patches: Locations are chosen to simulate real interactions for attentional control of visual processing: occipital, frontal right, temporal right, and posterior parietal left and right areas. (Right) The mean and standard deviation of the singular value of the lead field matrices for the randomly generated patches.

The number of eigenvector constraints used for each source patch in (10) was found through the singular values of randomly generated source patches on the cortex. The result in Fig. 1 (right) shows the 5th and higher singular values were small and we therefore used $l=4$.

The performance of minimum norm imaging, the standard LCMV beamformer, and the proposed nulling LCMV beamformer method, with both point and eigenvector constraints, is compared in the left column of Fig 2. The result shows the DTF between each pair of cortical regions computed from the signals estimated using each of the different spatial inverse methods. In these $5 \times 5$ plots, we show the DTF between each region pair as a function of frequency. As a qualitative indication of performance, we indicate in red those DTFs which should be zero, i.e. those regions between which there was no actual interaction when generating the simulated MVAR model. Consequently, DTFs that are red indicate errors in the estimated interactions.

The DTFs clearly indicate errors in the estimated interactions using the conventional LCMV beamformer and minimum norm imaging. The errors are somewhat smaller using the nulling beamformer with point constraints, but are substantially lower when using the more robust eigenvector constraints.

On the right side of Fig 2 we show the estimated connectivity as computed from the DTF's integrated from $12-30 \mathrm{~Hz}$ and thresholded at a $p=0.05$ significance level based on the permutation test. The new nulling LCMV method with eigenvector constraints shows the correct functional connectivity, while the other three methods show additional erroneous (red) connections.

The locations used to define the LCMV constraints were the true locations of the simulated sources. In real experiments, the actual locations are unknown and usually estimated by various source localization methods. Simulations we are unable to include here show that the eigenvector constraint method is robust to inaccurate source locations when the source location is mislocated by less than $5 \mathrm{~mm}$. 

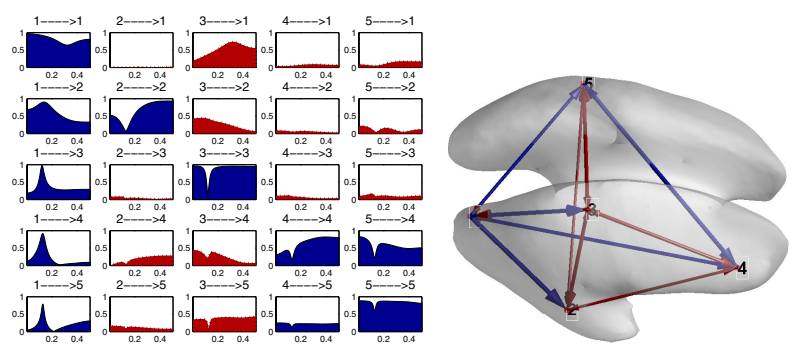

(a)LCMV beamformer with point constraints
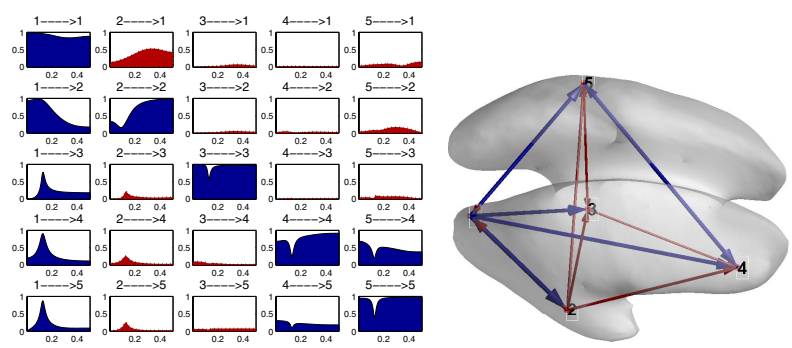

(b)Minimum norm method
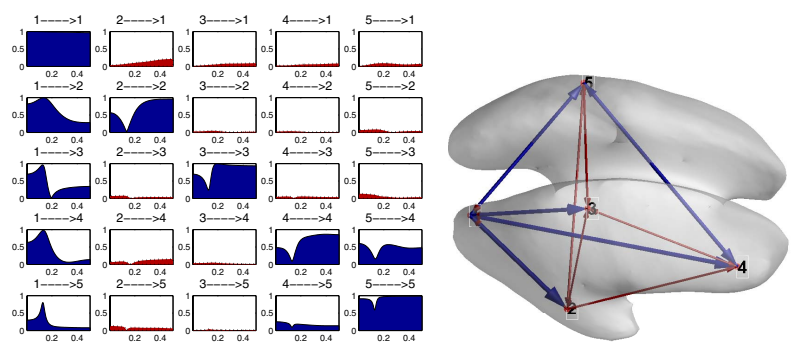

(c)LCMV nulling beamformer with point constraints
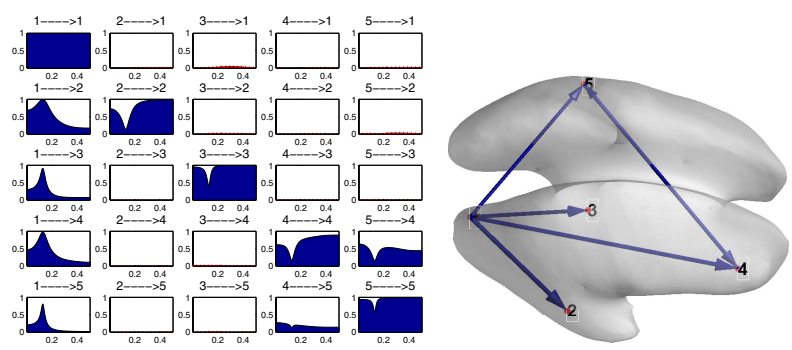

(d) LCMV nulling beamformer with eigenvector constraints

Fig. 2. Left: The DTF computed from (a) the estimated source signals by point constraint beamformer method, (b) the estimated source signals by minimum norm cortical imaging, (c) the estimated source signals by the new point constraint nulling beamformer method, and (d) the estimated source signals by eigenvector constraint nulling beamformer method. The horizontal axis represents the angular frequency from 0 to $0.5 \pi$, the vertical axis represents the DTF. Those with blue areas represent true interactions among the sources while those with red areas represent false interactions. Right: Permutation test of significance for functional connectivity based on the integrated DTF from estimated source signals. True interactions are shown in blue arrows and false in red. The radius of the arrows represents the strength of interaction.

\section{CONCLUSIONS}

We have demonstrated that the new eigenvector constrained nulling beamformer method is effective in simulated data for estimating source signals and suppressing cross-talk; the estimated MVAR parameters and DTF's accurately reflected functional connectivity. Our simulations also show some robustness to inaccurate estimation of the source locations. Finally, the permutation test appears successful in identifying significant interactions.

Although we have attempted to make the method robust through the use of eigenvector constraints and nulling, our approach shares with other beamforming methods a sensitivity to the assumed underlying model. In our case the primary sensitivity will be to additional correlated sources which contribute to the measured EEG/MEG data but are not included in the set of constraints. Sources of this type would certainly confound interpretation of cortical interactions using MVAR modeling, as would silent hidden sources. Nevertheless, the approach described does appear effective in addressing the cross-talk problem which make minimum norm imaging and conventional LCMV beamforming inappropriate for this application.

\section{References}

[1] M. Ding, S. L. Bressler, W. Yang, and H. Liang, "Short-window spectral analysis of cortical event-related potentials by adaptive multivariate autoregressive modeling: data preprocessing, model validation, and variability assessment," Biological cybernetics, vol. 83, no. 1, pp. 35-45, 2000.

[2] M. Kaminski and et al., "Evaluating causal relations in neural systems: granger causality, directed transfer function and statistical assessment of significance," Biol Cybern, vol. 85, no. 2, pp. 145-57, 2001.

[3] R. Kus and et al., "Determination of eeg activity propagation: pair-wise versus multichannel estimate," Biomedical Engineering, IEEE Transactions on, vol. 51, no. 9, pp. 1501-1510, 2004.

[4] Andrea Brovelli, Mingzhou Ding, Anders Ledberg, Yonghong Chen, Richard Nakamura, and Steven L. Bressler, "Beta oscillations in a large-scale sensorimotor cortical network: Directional influences revealed by granger causality," PNAS, vol. 101, no. 26, pp. 9849-9854, 2004.

[5] L. Astolfi and et al., "Estimation of the effective and functional human cortical connectivity with structural equation modeling and directed transfer function applied to high-resolution eeg," Magn Reson Imaging, vol. 22, no. 10, pp. 1457-1470, 2004.

[6] B.D. Van Veen and K.M. Buckley, "Beamforming: a versatile approach to spatial filtering," ASSP Magazine, IEEE, vol. 5, no. 2, pp. 4-24, 1988.

[7] B.D. Van Veen and et al., "Localization of brain electrical activity via linearly constrained minimum variance spatial filtering," Biomedical Engineering, IEEE Transactions on, vol. 44, no. 9, pp. 867-880, 1997.

[8] J. Gross and et al., "Modulation of long-range neural synchrony reflects temporal limitations of visual attention in humans," PNAS, vol. 101, no. 35, pp. 13050-13055, 2004. 